\title{
Delegation in a group-contest
}

\author{
Lambert Schoonbeek* \\ Department of Economics \\ University of Groningen
}

SOM-theme F: Interactions between consumers and firms

\begin{abstract}
It is known that the option of delegation in a rent-seeking contest between two individuals leads to a prisoner's dilemma. In equilibrium no player hires a delegate, while it is Pareto-efficient if both would hire one. This paper shows that this result does not generalize to a contest between the members of two groups. We further provide the conditions under which zero, one, or both groups hire a delegate in equilibrium.
\end{abstract}

Keywords: contests, rent-seeking, delegation

JEL-codes: D72, C72

\footnotetext{
* Address: Lambert Schoonbeek, Department of Economics, University of Groningen, P.O. Box 800, NL9700 AV Groningen, The Netherlands. Fax: +31 50363 7337, E-mail: L.Schoonbeek@eco.rug.nl. I thank Marco Haan, Joyce Jacobsen, and Peter Kooreman for helpful discussions.
} 


\section{Introduction}

In a seminal paper, Tullock (1980) introduced his rent-seeking contest in which two individuals compete for a given rent or prize. The prize is assigned to one of the players, and each player exerts effort to increase the probability that he will win it. The model is applied and extended in several directions, see e.g. Nitzan (1994) and Lockard and Tullock (2001). In particular, Wärneryd (2000) considered the situation in which each player can hire a delegate who acts on his behalf in the contest. He assumes that a player cannot observe the effort of his delegate. To deal with the moral hazard, the payment to the delegate must depend on the outcome of the contest. Assuming limited liability for the delegate, the player offers the delegate a contingent fee, i.e. the delegate gets a deliberately chosen fee in case of success and nothing otherwise. Wärneryd shows that there is a no-delegation prisoner's dilemma, i.e. no player voluntarily wants to hire a delegate, although both are better off if both would hire one. Other studies on delegation in standard two-person rent-seeking contests are Baik and Kim (1997) and Schoonbeek (2002), which focus on, respectively, different abilities and risk-attitudes of players and their delegates.

In the present paper, we analyze delegation in a rent-seeking group-contest as presented by Nitzan (1991a). In this contest, the players are the members of two interest groups and the prize is awarded to one of these groups. The probability that a groups gets the prize depends on the relative size of the aggregate group efforts. The prize exhibits private good characteristics, and if it is won by a group, it is divided among its members. Nitzan(1991a) assumes that the prize is distributed either equally among the members, or in proportion to each members' effort, or more generally, by using a given mixture of these principles. For analytical simplicity, and to get clear-cut results, we focus here on the equal-sharing rule. Examples of this kind of contest are group-contests by interest- or lobbying groups (parties, departments, industries, etc.) for budgets at the discretion of policy makers. See further also Baik and Lee (1997), and Nitzan (1991b).

We remark now that, in practice, groups are often represented by a delegate. Motivated by this, the present paper extends the group-contest by giving each group the option to hire a delegate who acts on its behalf in the contest. Following Wärneryd (2000), we assume that a delegate is offered a contingent fee contract. In section 2 we discuss the model and give some preliminary results. Next, in section 3 we show that, in contrast with the contest between two individuals, groups might hire a delegate in the Nash equilibrium. In particular, we provide the conditions, in terms of the number of members in each group, under which in equilibrium zero, one or both groups hire a delegate. We further show that the no-delegation prisoner's dilemma result of Wärneryd for the contest between two individuals also does not generalize to the group contest. In particular, if both groups are equally large, then in equilibrium both groups will not hire a delegate if each has 2, 3 , 4, 5 or 6 members. However, in those cases the equilibrium is Pareto efficient. Further, if both groups are equally large and each has at least 7 members, then in equilibrium both groups $d o$ hire a delegate. In the latter cases we have a 'reversed' prisoner's dilemma, as then the members of both groups would benefit if both groups would not hire a delegate. We conclude in section 4. 


\section{The model and preliminary results}

Consider a contest between interest groups 1 and 2. Group $i(i=1,2)$ consists of $n_{i} \geq 1$ risk-neutral members. The members of both groups compete for a prize of value $S>0$, which will be awarded to one of the groups. We assume that if group $i$ wins the prize, each member gets $S / n_{i}$ of it. The contest is a two-stage game. In stage 1, each group decides whether or not it will hire a risk-neutral delegate who will represent this group as a contestant in stage 2 . If a group hires no delegate, then in stage 2 its members will be the contestants themselves. In stage 2, the relevant contestants compete for the prize by voluntarily exerting efforts. Following the rent-seeking literature, we assume that these contestants observe each other's efforts. The probability, $p$ say, that group 1 wins the prize depends on the relative size of the efforts of the contestants in stage 2 . The probability that group 2 wins the prize equals $1-p$.

If group $i(i=1,2)$ wants to hire a delegate it offers the delegate a contract that specifies his payment. There is moral hazard as the members of group $i$ are not able to monitor the effort of their delegate in stage 2 . Thus, the payment cannot be made dependent on this effort. Instead, the delegate is offered a contingent fee contract: he gets a deliberately chosen fee $w_{i}>0$ if group $i$ wins the prize and nothing otherwise. As the counterpart of the equal division of the prize, we assume that each member of group $i$ has to contribute an equal share of $w_{i}$. We further assume that a delegate accepts the offered contract if his expected payoff under the contract is nonnegative.

We derive the (subgame-perfect Nash) equilibrium in pure strategies of the contest. Using backward induction, we distinguish four possible cases in stage 2: both groups hire no delegate (case a), only group 2 hires a delegate (case b), only group 1 hires a delegate (case c), and both groups hire a delegate (case d). If the members of group $i(i=1,2)$ compete themselves in stage 2 , let $x_{k i}$ denote the effort of member $k\left(k=1, \ldots, n_{i}\right)$, and $x_{i} \equiv$ $\sum_{k=1}^{n_{i}} x_{k i}$ denote group $i$ 's total effort. If group $i(i=1,2)$ hires a delegate, let $y_{i}$ denote the effort of this delegate in stage 2. We now consider the candidate equilibria associated with the cases a) to d) in more detail. For details of the derivations of the formulas of part b) and d) we refer to Appendix B.

- Case a: In stage 2 the contestants are the members of group 1 and 2. Following Nitzan (1991a), we assume that the probability that group 1 wins the prize is given by $p=$ $x_{1} /\left(x_{1}+x_{2}\right)$ if $x_{1}+x_{2}>0$ and $p=1 / 2$ if $x_{1}+x_{2}=0$. Member $k\left(k=1, \ldots, n_{i}\right)$ of group $i(i=1,2)$ maximizes his own expected payoff, i.e. he solves the problem

$$
\max _{x_{k i}}\left(\frac{x_{i}}{x_{i}+x_{j}}\right) \frac{S}{n_{i}}-x_{k i}
$$

where $j \neq i$. It can be verified that in the (symmetric) candidate equilibrium, the winningprobability of group 1 is $p^{a}=n_{2} /\left(n_{1}+n_{2}\right)$, while the effort of member $k$ of group $i$ is

$$
x_{k i}^{a}=\frac{n_{j} S}{n_{i}\left(n_{i}+n_{j}\right)^{2}}
$$


and the corresponding expected payoff of member $k$ of group $i$ equals

$$
\pi_{k i}^{a}=\frac{n_{j}\left(n_{i}+n_{j}-1\right) S}{n_{i}\left(n_{i}+n_{j}\right)^{2}}
$$

We remark that (2) and (3) correspond to the equilibrium effort and payoff in the group contest without delegation of Nitzan (1991a, p. 1526). As noticed by Nitzan, if the size of a group increases, the members of that group have incentives to reduce their efforts. The reason is that there are free-riding effects within the group in the competition for the prize, as well as group-size prize-effects, i.e. an increase in the group size reduces each members' share of the prize. As a result, the larger the group size, the lower the efforts and expected payoffs, see (2) and (3).

- Case $b$ : In stage 2 the contestants are the members of group 1 and the delegate of group 2. The probability that group 1 wins the prize is given by $p=x_{1} /\left(x_{1}+y_{2}\right)$ if $x_{1}+y_{2}>0$ and $p=1 / 2$ if $x_{1}+y_{2}=0$. Maximizing expected payoff, member $k\left(k=1, \ldots, n_{1}\right)$ of group 1 solves

$$
\max _{x_{k 1}}\left(\frac{x_{1}}{x_{1}+y_{2}}\right) \frac{S}{n_{1}}-x_{k 1}
$$

while, given $w_{2}$, the delegate of group 2 solves

$$
\max _{y_{2}}\left(\frac{y_{2}}{x_{1}+y_{2}}\right) w_{2}-y_{2}
$$

One can check that in the (symmetric) candidate equilibrium the efforts are given by

$$
\begin{aligned}
x_{k 1} & =\frac{\left(\frac{S}{n_{1}}\right)^{2} w_{2}}{n_{1}\left(\frac{S}{n_{1}}+w_{2}\right)^{2}}, \quad k=1, \ldots, n_{1} \\
y_{2} & =\frac{\left(\frac{S}{n_{1}}\right) w_{2}^{2}}{\left(\frac{S}{n_{1}}+w_{2}\right)^{2}}
\end{aligned}
$$

Next, observing that $p=\left(S / n_{1}\right) /\left(\left(S / n_{1}\right)+w_{2}\right)$, group 2 maximizes expected payoff in stage 1 by offering the delegate in case of success a fee $w_{2}$ that solves

$$
\max _{w_{2}}\left(\frac{w_{2}}{\frac{S}{n_{1}}+w_{2}}\right)\left(\frac{S-w_{2}}{n_{2}}\right)
$$

The optimal fee turns out to equal $w_{2}^{b}=\left(-1+\sqrt{1+n_{1}}\right) S / n_{1}$. Substituting this in (6) and (7), we derive that in the candidate equilibrium the winning-probability of group 1 is $p^{b}=1 / \sqrt{1+n_{1}}$, while the expected payoffs of the members of group 1 and 2 are equal to

$$
\begin{aligned}
& \pi_{k 1}^{b}=\frac{\left(\left(n_{1}-1\right) \sqrt{1+n_{1}}+1\right) S}{n_{1}^{2}\left(1+n_{1}\right)}, \quad k=1, \ldots, n_{1} \\
& \pi_{k 2}^{b}=\frac{\left(1+n_{1}-\sqrt{1+n_{1}}\right)^{2} S}{n_{1} n_{2}\left(1+n_{1}\right)}, \quad k=1, \ldots, n_{2}
\end{aligned}
$$

- Case c: The results for case c) directly follow from case b) by interchanging the indices 
1 and 2. In particular, in the (symmetric) candidate equilibrium the winning-probability of group 1 is $p^{c}=\left(-1+\sqrt{1+n_{2}}\right) / \sqrt{1+n_{2}}$, while the expected payoffs of the members of the groups 1 and 2 are equal to

$$
\begin{aligned}
& \pi_{k 1}^{c}=\frac{\left(1+n_{2}-\sqrt{1+n_{2}}\right)^{2} S}{n_{1} n_{2}\left(1+n_{2}\right)}, \quad k=1, \ldots, n_{1} \\
& \pi_{k 2}^{c}=\frac{\left(\left(n_{2}-1\right) \sqrt{1+n_{2}}+1\right) S}{n_{2}^{2}\left(1+n_{2}\right)}, \quad k=1, \ldots, n_{2}
\end{aligned}
$$

- Case $d$ : In stage 2 the two delegates compete with each other. The probability that group 1 wins the prize is given by $p=y_{1} /\left(y_{1}+y_{2}\right)$ if $y_{1}+y_{2}>0$ and $p=1 / 2$ if $y_{1}+y_{2}=0$. Maximizing expected payoff, delegate $i(i=1,2)$ of group $i$ solves, given $w_{i}$,

$$
\max _{y_{i}}\left(\frac{y_{i}}{y_{i}+y_{j}}\right) w_{i}-y_{i}
$$

where $j \neq i$. The corresponding effort of delegate $i$ is $y_{i}=w_{i}^{2} w_{j} /\left(w_{i}+w_{j}\right)^{2}$ Next, observing that $p=w_{1} /\left(w_{1}+w_{2}\right)$, group $i(i=1,2)$ maximizes expected payoff in stage 1 by solving

$$
\max _{w_{i}}\left(\frac{w_{i}}{w_{i}+w_{j}}\right)\left(\frac{S-w_{i}}{n_{i}}\right)
$$

Hence, in the candidate equilibrium the fee in case of success for delegate $i$ equals $w_{i}^{d}=$ $S / 3$, while the winning-probability of group 1 is $p^{d}=1 / 2$, and the expected payoff of member $k$ of group $i$ is

$$
\pi_{k i}^{d}=\frac{S}{3 n_{i}}, \quad k=1, \ldots, n_{i}
$$

\section{Delegation in equilibrium}

Next, we examine whether in equilibrium the groups will hire a delegate. Using (3), (9), (10), (11), (12) and (15), we first present two useful lemmas.

Lemma 3.1 For all $n_{1} \geq 1$ and $n_{2} \geq 1$, and all relevant $k$ we have:

(i) $\pi_{k 1}^{b}>\pi_{k 1}^{d}$ if $n_{1} \leq 6$, and $\pi_{k 1}^{b}<\pi_{k 1}^{d}$ if $n_{1} \geq 7$.

(ii) $\pi_{k 2}^{c}>\pi_{k 2}^{d}$ if $n_{2} \leq 6$, and $\pi_{k 2}^{c}<\pi_{k 2}^{d}$ if $n_{2} \geq 7$.

Proof: See Appendix A.

Without loss of generality we may order the groups such that $n_{1} \geq n_{2} \geq 1$. Doing so, we have:

Lemma 3.2 Assume that $n_{1} \geq n_{2} \geq 1$. For all relevant $k$ we have: 
(i) Let $n_{2}=1$. Then $\pi_{k 1}^{a}>\pi_{k 1}^{c}$ if $n_{1} \leq 3$, and $\pi_{k 1}^{a}<\pi_{k 1}^{c}$ if $n_{1} \geq 4$.

(ii) Let $n_{2}=2$, 3. Then $\pi_{k 1}^{a}>\pi_{k 1}^{c}$ if $n_{1} \leq 4$, and $\pi_{k 1}^{a}<\pi_{k 1}^{c}$ if $n_{1} \geq 5$.

(iii) Let $n_{2}=4$, 5. Then $\pi_{k 1}^{a}>\pi_{k 1}^{c}$ if $n_{1} \leq 5$, and $\pi_{k 1}^{a}<\pi_{k 1}^{c}$ if $n_{1} \geq 6$.

(iv) Let $n_{2}=6$. Then $\pi_{k 1}^{a}>\pi_{k 1}^{c}$ if $n_{1}=6$, and $\pi_{k 1}^{a}<\pi_{k 1}^{c}$ if $n_{1} \geq 7$.

(v) Let $n_{2} \geq 7$. Then $\pi_{k 1}^{a}<\pi_{k 1}^{c}$.

(vi) Let $n_{2} \leq 6$. Then $\pi_{k 2}^{a}>\pi_{k 2}^{b}$.

\section{Proof: See Appendix A.}

For notational convenience, let (d,nd) denote the case in which group 1 hires a delegate and group 2 hires no delegate. In a similar way, we define (nd,d), (nd,nd) and (d,d). We then present the following result:

Proposition 3.1 Assume that $n_{1} \geq n_{2} \geq 1$. In equilibrium the groups decide in stage 1 as follows whether they hire or not a delegate:

(i) Let $n_{2}=1$. Then the groups choose (nd,nd) if $n_{1} \leq 3$, and (d,nd) if $n_{1} \geq 4$.

(ii) Let $n_{2}=2,3$. Then the groups choose ( $\left.n d, n d\right)$ if $n_{1} \leq 4$, and (d,nd) if $n_{1} \geq 5$.

(iii) Let $n_{2}=4,5$. Then the groups choose (nd,nd) if $n_{1} \leq 5$, and (d,nd) if $n_{1} \geq 6$.

(iv) Let $n_{2}=6$. Then the groups choose (nd,nd) if $n_{1}=6$, and (d,nd) if $n_{1} \geq 7$.

(v) Let $n_{2} \geq 7$. Then the groups choose $(d, d)$.

\section{Proof: See Appendix A.}

Table 3.1 gives an overview of the results of Proposition 3.1 for $1 \leq n_{2} \leq n_{1} \leq 8$. Assuming that group 2 is not larger than group 1, we see from (i) to (iv) of Proposition 3.1 that group 2 does not hire a delegate if $n_{2}$ is 'small' (i.e. $n_{2} \leq 6$ ). On the other hand, in that case the decision of group 1 depends on the relative size of $n_{1}$ versus $n_{2}$ : group 1 hires a delegate if and only if its size is 'relatively large enough'. Next, (v) of Proposition 3.1 shows that both groups will hire a delegate if both are 'large' (i.e. $n_{1} \geq n_{2} \geq 7$ ).

Table 3.1: Illustration of Proposition 3.1

\begin{tabular}{|l|c|cccccccc|}
\hline & & \multicolumn{7}{|c|}{$n_{2}$} \\
\hline & & 1 & 2 & 3 & 4 & 5 & 6 & 7 & 8 \\
\hline \multirow{5}{*}{$n_{1}$} & 1 & (nd,nd) & - & - & - & - & - & - & - \\
& 2 & (nd,nd) & (nd,nd) & - & - & - & - & - & - \\
& 3 & (nd,nd) & (nd,nd) & (nd,nd) & - & - & - & - & - \\
& 4 & (d,nd) & (nd,nd) & (nd,nd) & (nd,nd) & - & - & - & - \\
& 5 & (d,nd) & (d,nd) & (d,nd) & (nd,nd) & (nd,nd) & - & - & - \\
& 6 & (d,nd) & (d,nd) & (d,nd) & (d,nd) & (d,nd) & (nd,nd) & - & - \\
& 7 & (d,nd) & (d,nd) & (d,nd) & (d,nd) & (d,nd) & (d,nd) & (d,d) & - \\
& 8 & (d,nd) & (d,nd) & (d,nd) & (d,nd) & (d,nd) & (d,nd) & (d,d) & (d,d) \\
\hline
\end{tabular}


To understand Proposition 3.1, first recall the free-riding effects and group-size prizeeffects that arise in the group-contest without delegation of Nitzan (1991a) [see our discussion of case a) in section 2]. Next, examine the option of delegation, and consider group 1 say. If group 1 hires a delegate, there are a number of effects. First, considering stage 2, group 1 is represented now by one delegate instead of $n_{1}$ individuals. This removes freerider effects related to group 1 in stage 2 . Further, if $n_{1}$ is large enough, then the stake of the delegate is larger than the stake of each member of group 1 in the case of no delegation [i.e., comparing cases c) and a) we have $w_{1}^{c} \geq S / n_{1}$, and comparing cases d) and b) we have $w_{1}^{d} \geq S / n_{1}$, if $n_{1}$ is large enough]. All this leads to a positive effect of delegation for group 1, i.e larger efforts in favour of group 1 and thus a higher probability that it will win the prize [i.e., if $n_{1}$ is large enough, then $p^{c}>p^{a}$ and $p^{d}>p^{b}$ ].

Second, turning to stage 1 , we see that there is a negative effect of delegation because the net prize of each member of group 1 decreases as each one has to contribute to the fee of the delegate in case of success. On the other hand, if group 1 hires a delegate but does not win the prize, its members incur no costs as all effort expenditures are born by their delegate. Summarizing, intuitively speaking, Proposition 3.1 shows that if $n_{1}$ is large enough, then the positive effects of stage 1 and 2 dominate, and as a result, group 1 hires a delegate. More generally, Proposition 3.1 demonstrates that in contrast with the standard contest between two individuals, in a group contest there are conditions such that one or both groups hire a delegate.

Next, we will show that the no-delegation prisoner's dilemma result of Wärneryd (2000) for the standard contest between two individuals also does not generalize to the group contest. To do so, we focus on the situation in which both groups have an equal size.

Proposition 3.2 Suppose that $n_{1}=n_{2}=n \geq 1$. The following holds:

(i) If $1 \leq n \leq 6$, then irrespective as to whether the other group hires a delegate, for each group it is optimal not to hire a delegate in stage 1 . If $n \geq 7$, then irrespective as to whether the other group hires a delegate, for each group it is optimal to hire a delegate in stage 1.

(ii) Let $n=1$. Then in equilibrium ( $(n d, n d)$ is the outcome of stage 1. However, if $(d, d)$ would be chosen in stage 1 [i.e. the groups would behave according to case d) of section 2], this would give a Pareto improvement over the equilibrium.

(iii) Let $2 \leq n \leq 6$. Then in equilibrium ( $n d, n d)$ is the outcome of stage 1. Moreover, the equilibrium is Pareto efficient.

(iv) Let $n \geq 7$. Then in equilibrium $(d, d)$ is the outcome of stage 1. However, if ( $n d, n d)$ would be chosen in stage 1 [i.e. the groups would behave according to case a) of section 2], this would give a Pareto improvement over the equilibrium.

Proof: See Appendix A.

Discussing Proposition 3.2, first consider part (ii). Noting that in this case both groups have just one member, and combining it with part (i), we see that the (nd,nd) outcome cor- 
responds to the prisoner's dilemma analysed by Wärneryd (2000). Second, part (iii) shows that if both groups have 2, 3, 4, 5 or 6 members, they still choose (nd,nd) in equilibrium. It is interesting to observe, however, that now this outcome is Pareto efficient! We no longer have a prisoner's dilemma. Third, combining part (iv) with part (i), if both groups have 7 or more members, we again have a prisoner's dilemma. However, now the dilemma is 'reversed', i.e. both groups hire a delegate, while both would benefit if both would not hire one!

In order to understand the Pareto (in)efficiency results, notice first that in the symmetric case considered in Proposition 3.2, in equilibrium each group always wins the rent with probability 0.5 , irrespective as to whether both groups hire a delegate or not. Next, if both groups hire a delegate, then the 'total cost' for both groups together is equal to the fee that must be paid to the delegate of the group that wins the prize. This fee is equal to $S / 3$, see case d) in section 2. On the other hand, if both groups do not hire a delegate, then the 'total cost' of both groups together is given by the summation of all their efforts. Using (2), it follows that the latter is equal to $S / 2 n$. We observe now that $S / 3<S / 2 n$ if $n=1$, which explains the inefficiency result of (ii) of Proposition 3.2. Further, $S / 3>S / 2 n$ for all $n \geq 2$, which explains the (in)efficiency results of (iii) and (iv) of Proposition 3.2.

\section{Concluding remarks}

We have analysed the option of delegation in a contest between the members of two interest groups. As known, in the case without delegation there are free-rider effects and groupsize prize-effects, which give the members of a group an incentive to reduce their efforts if their group-size increases. We have shown that if we introduce the option of delegation, then depending on the relative size of the groups, in equilibrium zero, one, or both groups decide to hire a delegate. If a group is large enough, then by hiring a delegate, the group mitigates the free-rider- and group-size prize-effects, which results in more aggressive behaviour on behalf of the group, and in turn in a higher probability that it will win the prize, and larger expected payoffs.

Finally, recall that we have assumed that the prize is distributed using the equal sharingrule. Nitzan (1991a) also considers the general case in which a given part of the prize is distributed with the equal-sharing rule and the remaining part in proportion to the relative efforts of the members of the winning group. He argues that (in the contest without the option of delegation) in equilibrium the mentioned free-rider- and group-size prize-effects become smaller the more the prize is distributed according to relative efforts. Hence, we might expect that in that case the incentive to hire a delegate becomes smaller as well. 


\section{References}

Baik, K.H. and I.-G. Kim (1997), "Delegation in contests", European Journal of Political Economy, 13, 281-298.

Baik, K.H. and S. Lee (1997), "Collective rent seeking with endogenous group sizes", European Journal of Political Economy, 13, 121-130.

Lockard, A.L. and G. Tullock, editors (2001), Efficient rent-seeking: chronicle of an intellectual quagmire, Kluwer Academic Publishers, Boston.

Nitzan, S. (1991a), "Collective rent dissipation", Economic Journal, 101, 1522-1534.

Nitzan, S. (1991b), "Rent-seeking with non-identical sharing rules", Public Choice, 71, 43-50.

Nitzan, S. (1994), "Modelling rent-seeking contests", European Journal of Political Economy, 10, 41-60.

Schoonbeek, L. (2002), "A delegated agent in a winner-take-all contest", Applied Economics Letters, 9, 21-23.

Tullock, G. (1980), "Efficient rent seeking", in J.M. Buchanan, R.D. Tollison, and G. Tullock, editors, Toward a theory of the rent-seeking society, College Station: Texas A\&M University Press, 97-112.

Wärneryd, K. (2000), "In defense of lawyers: moral hazard as an aid to cooperation", Games and Economic Behavior, 33, 145-158. 


\section{Appendix A}

Proof of Lemma 3.1: Using (9) and (15), we have $\pi_{k 1}^{b}>\pi_{k 1}^{d}$ if and only if $3+3\left(n_{1}-\right.$ 1) $\sqrt{1+n_{1}}>n_{1}\left(1+n_{1}\right)$. One can verify that $3+3\left(n_{1}-1\right) \sqrt{1+n_{1}}>n_{1}\left(1+n_{1}\right)$ if $1 \leq n_{1} \leq 6$, whereas $3+3\left(n_{1}-1\right) \sqrt{1+n_{1}}<n_{1}\left(1+n_{1}\right)$ if $n_{1} \leq 7$, which proves part (i) of Lemma 3.1. Part (ii) follows similarly.

Proof of Lemma 3.2: To begin with, define the auxiliary real-valued functions $f(s, t) \equiv$ $(s+t-1) /(s+t)^{2}$, and $g(u) \equiv(\sqrt{1+u}-1)^{2} / u^{2}$, where $s, t, u \geq 1$. We make three observations. First, $\partial f(s, t) / \partial s<0$ and $\partial f(s, t) / \partial t<0$ if $s+t>2$. Second, taking $s=t=u$, we have $f(s, s)>g(s)$ if and only if $2 s^{2}+11 s+9-8(1+s) \sqrt{1+s}<0$. Using this, one can verify that $f(s, s)>g(s)$ if $1 \leq s \leq 6$, whereas $f(s, s)<g(s)$ if $s \geq 7$. Third, $f(s, 6)>g(s)$ if and only if $7 s^{2}+36 s+2(s+6)^{2}(1-\sqrt{1+s})<0$. Using this, one can show that $f(s, 6)>g(s)$ if $s \geq 6$. Using these observations, we first prove parts (i)-(v), and next part (vi).

- Parts (i)-(v): Using (3) and (11), we have $\pi_{k 1}^{a}<(>) \pi_{k 1}^{c}$ if and only if $f\left(n_{1}, n_{2}\right)<(>$ ) $g\left(n_{2}\right)$. Let us examine now situations with a given $n_{2} \geq 7$. If $n_{1}$ satisfies $n_{1}=n_{2}$, we know that $f\left(n_{1}, n_{2}\right)=f\left(n_{2}, n_{2}\right)<g\left(n_{2}\right)$. If we next increase $n_{1}$, keeping $n_{2}$ fixed, we have $f\left(n_{1}, n_{2}\right)<g\left(n_{2}\right)$ for all $n_{1} \geq n_{2}$, since the partial derivative of $f($.$) with respect$ to its first argument is negative $\left(n_{1}+n_{2}>2\right)$. Part (v) follows directly. Next, examine situations with a given $n_{2}$, where $1 \leq n_{2} \leq 6$. If $n_{1}$ satisfies $n_{1}=n_{2}$, we know that $f\left(n_{1}, n_{2}\right)=f\left(n_{2}, n_{2}\right)>g\left(n_{2}\right)$. If we next increase $n_{1}$, keeping $n_{2}$ fixed, we have that $f\left(n_{1}, n_{2}\right)<g\left(n_{2}\right)$ for $n_{1}$ 'large enough'. In particular, straightforward calculations show that if $n_{2}=1$, then $f\left(n_{1}, n_{2}\right)>g\left(n_{2}\right)$ for $n_{1} \leq 3$, whereas $f\left(n_{1}, n_{2}\right)<g\left(n_{2}\right)$ for $n_{1} \geq 4$. Part (i) follows directly. The parts (ii), (iii) and (iv) follow similarly.

- Part (vi): Using (3) and (10), we have $\pi_{k 2}^{a}>\pi_{k 2}^{b}$ if and only if $f\left(n_{1}, n_{2}\right)>g\left(n_{1}\right)$. We distinguish now the following cases: $(\alpha) 1 \leq n_{2}=n_{1} \leq 5,(\beta) 1 \leq n_{2}<n_{1} \leq 5$, and $(\gamma)$ $1 \leq n_{2} \leq 6$ ànd $n_{1} \geq 6$. We know, see above, that $f\left(n_{1}, n_{2}\right)>g\left(n_{1}\right)$ in case $(\alpha)$. Straightforward calculations show that $f\left(n_{1}, n_{2}\right)>g\left(n_{1}\right)$ as well in case $(\beta)$. Finally, because the partial derivative of $f$ (.) with respect to its second argument is negative $\left(n_{1}+n_{2}>2\right)$, it follows that $f\left(n_{1}, n_{2}\right)>g\left(n_{1}\right)$ in case $(\gamma)$ if the condition $f\left(n_{1}, 6\right)>g\left(n_{1}\right)$ holds for all $n_{1} \geq 6$. We know that the latter condition holds, see above.

Proof of Proposition 3.1: Suppose that $n_{2}=1$ and $n_{1} \leq 3$. We then have from (i) of Lemma 3.1 that $\pi_{k 1}^{b}>\pi_{k 1}^{d}$, and from (i) of Lemma 3.2 that $\pi_{k 1}^{a}>\pi_{k 1}^{c}$. Hence, group 1 does not hire a delegate. Using (vi) of Lemma 3.2, we have $\pi_{k 2}^{a}>\pi_{k 2}^{b}$. Combining results, (nd,nd) is the equilibrium outcome in stage 1 . Next, suppose that $n_{2}=1$ and $n_{1} \geq 4$. It then follows from (ii) of Lemma 3.1 that $\pi_{k 2}^{c}>\pi_{k 2}^{d}$, and from (vi) of Lemma 3.2 that $\pi_{k 2}^{a}>\pi_{k 2}^{b}$. So, group 2 does not hire a delegate. Using (i) of Lemma 3.2, we have $\pi_{k 1}^{a}<\pi_{k 1}^{c}$. Combining results, $(\mathrm{d}, \mathrm{nd})$ is the equilibrium outcome in stage 1 . This establishes part (i) of the proposition. The other parts follow similarly. 
Proof of Proposition 3.2: Taking $1 \leq n \leq 6$, we obtain from (ii) of Lemma 3.1 that $\pi_{k 2}^{c}>\pi_{k 2}^{d}$ and from (vi) of Lemma 3.2 that $\pi_{k 2}^{a}>\pi_{k 2}^{b}$. Thus, in stage 1 'no delegate' is optimal for group 2. Using symmetry, the same applies to group 1 . Next, taking $n \geq 7$, (i) of Lemma 3.1 implies $\pi_{k 1}^{b}<\pi_{k 1}^{d}$, and (v) of Lemma 3.2 gives $\pi_{k 1}^{a}<\pi_{k 1}^{c}$. As a result, in stage 1 'delegate' is optimal for firm 1 . Using symmetry, the same applies to group 2 . This establishes part (i) of Proposition 3.2. The parts (ii), (iii) and (iv) then follow from part (i) of Proposition 3.2 and the observation that (3) and (15) imply that $\pi_{k i}^{a}<\pi_{k i}^{d}$ if $n=1$, and $\pi_{k i}^{a}>\pi_{k i}^{d}$ if $n \geq 2\left(i=1,2\right.$ and $\left.k=1, \ldots, n_{i}\right)$. 


\section{Appendix B}

Derivation of formulas of case $b$ )

First consider stage 2 . In this stage, member $k\left(k=1, \ldots, n_{1}\right)$ of group 1 solves

$$
\max _{x_{k 1}}\left(\frac{x_{1}}{x_{1}+y_{2}}\right) \frac{S}{n_{1}}-x_{k 1}
$$

while, given $w_{2}$, the delegate of group 2 solves

$$
\max _{y_{2}}\left(\frac{y_{2}}{x_{1}+y_{2}}\right) w_{2}-y_{2}
$$

The first-order condition for member $k$ of group 1 is

$$
\frac{y_{2}\left(\frac{S}{n_{1}}\right)}{\left(x_{1}+y_{2}\right)^{2}}=1
$$

and the first-order condition for the delegate of group 2 is

$$
\frac{x_{1} w_{2}}{\left(x_{1}+y_{2}\right)^{2}}=1
$$

Using these conditions we obtain $x_{1} w_{2}=y_{2} S / n_{1}$, and, in turn,

$$
\begin{aligned}
x_{k 1} & =\frac{\left(\frac{S}{n_{1}}\right)^{2} w_{2}}{n_{1}\left(\frac{S}{n_{1}}+w_{2}\right)^{2}}, \quad k=1, \ldots, n_{1} \\
y_{2} & =\frac{\left(\frac{S}{n_{1}}\right) w_{2}^{2}}{\left(\frac{S}{n_{1}}+w_{2}\right)^{2}}
\end{aligned}
$$

Compare with (6) and (7). Using these expressions, we further derive that

$$
p=\frac{\frac{S}{n_{1}}}{\left(\frac{S}{n_{1}}+w_{2}\right)}
$$

Next, consider stage 1 . Group 2 now solves the problem

$$
\max _{w_{2}}\left(\frac{w_{2}}{\frac{S}{n_{1}}+w_{2}}\right)\left(\frac{S-w_{2}}{n_{2}}\right)
$$

The corresponding first-order condition can be written as

$$
w_{2}^{2}+2 w_{2}\left(\frac{S}{n_{1}}\right)-\frac{S^{2}}{n_{1}}=0
$$

From this we derive that the fee in case of success equals

$$
w_{2}^{b}=\frac{\left(-1+\sqrt{1+n_{1}}\right) S}{n_{1}}
$$


As a result, the probability that group 1 wins the prize becomes

$$
p^{b}=\frac{1}{\sqrt{1+n_{1}}}
$$

while the effort of member $k$ of group 1 is

$$
x_{k 1}^{b}=\frac{\left(-1+\sqrt{1+n_{1}}\right) S}{n_{1}^{2}\left(1+n_{1}\right)}, \quad k=1, \ldots, n_{1}
$$

and the corresponding payoff is

$$
\pi_{k 1}^{b}=\frac{\left(\left(n_{1}-1\right) \sqrt{1+n_{1}}+1\right) S}{n_{1}^{2}\left(1+n_{1}\right)}, \quad k=1, \ldots, n_{1}
$$

Compare with (9). Furthermore, the effort of the delegate of group 2 is

$$
y_{2}^{b}=\frac{\left(-1+\sqrt{1+n_{1}}\right)^{2} S^{3}}{n_{1}^{3}\left(1+n_{1}\right)}
$$

and using this, the payoff of member $k$ of group 2 becomes

$$
\pi_{k 2}^{b}=\frac{\left(1+n_{1}-\sqrt{1+n_{1}}\right)^{2} S}{n_{1} n_{2}\left(1+n_{1}\right)}, \quad k=1, \ldots, n_{2}
$$

Compare with (10).

Derivation of formulas of case d)

First consider stage 2. Given the fees $w_{1}$ and $w_{2}$, the delegate of group 1 solves

$$
\max _{y_{1}}\left(\frac{y_{1}}{y_{1}+y_{2}}\right) w_{1}-y_{1}
$$

while the delegate of group 2 solves

$$
\max _{y_{2}}\left(\frac{y_{2}}{y_{1}+y_{2}}\right) w_{2}-y_{2}
$$

The corresponding first-order conditions are

$$
\begin{aligned}
& \frac{y_{2} w_{1}}{\left(y_{1}+y_{2}\right)^{2}}=1 \\
& \frac{y_{1} w_{2}}{\left(y_{1}+y_{2}\right)^{2}}=1
\end{aligned}
$$

From this, we obtain $y_{2}=y_{1} w_{2} / w_{1}$, and, in turn, that

$$
\begin{aligned}
& y_{1}=\frac{w_{1}^{2} w_{2}}{\left(w_{1}+w_{2}\right)^{2}} \\
& y_{2}=\frac{w_{1} w_{2}^{2}}{\left(w_{1}+w_{2}\right)^{2}}
\end{aligned}
$$


As a result

$$
p=\frac{w_{1}}{\left(w_{1}+w_{2}\right)}
$$

Next, consider stage 1 . Then member $k\left(k=1, \ldots, n_{1}\right)$ of group 1 solves

$$
\max _{w_{1}}\left(\frac{w_{1}}{w_{1}+w_{2}}\right)\left(\frac{S-w_{1}}{n_{1}}\right)
$$

The corresponding first-order condition is

$$
w_{1}^{2}+2 w_{1} w_{2}-S w_{2}=0
$$

Similarly, member $k\left(k=1, \ldots, n_{2}\right)$ of group 2 solves

$$
\max _{w_{2}}\left(\frac{w_{2}}{w_{1}+w_{2}}\right)\left(\frac{S-w_{2}}{n_{2}}\right)
$$

with corresponding first-order condition

$$
w_{2}^{2}+2 w_{1} w_{2}-S w_{1}=0
$$

Using these first-order conditions, one can verify that the fees are given by

$$
w_{1}^{d}=w_{2}^{d}=\frac{S}{3}
$$

As a result the probability that group 1 wins the prize equals $p^{d}=1 / 2$ and the expected payoff of member $k$ of group 1 is

$$
\pi_{k 1}^{d}=\frac{S}{3 n_{1}}, \quad k=1, \ldots, n_{1}
$$

while the corresponding expected payoff of member $k$ of group 2 is

$$
\pi_{k 2}^{d}=\frac{S}{3 n_{2}}, \quad k=1, \ldots, n_{2}
$$

Compare with (15). 